\title{
As possíveis causas da não adesão à imunização no Brasil: uma revisão de literatura
}

\author{
Possible causes of non-adherence to immunization in Brazil: a literature review
}

Posibles causas de incumplimiento de la vacunación en Brasil: revisión de la literatura

Shesllen Mikaelly Cruz Corrêa ${ }^{1 *}$, Pedro Fonseca de Vasconcelos ${ }^{1}$, Jamille Santos dos Passos ${ }^{1}$, Valéria Gomes Marques ${ }^{1}$, Nadynne Porto Magalhães Tanajura1, Davi Ramos do Nacimento1, Camila Santana Silva ${ }^{1}$, Laila Pereira Magalhães ${ }^{1}$, Lilian Rhodes Neves ${ }^{2}$, Michelle Beatriz Santos Silveira ${ }^{3}$.

\section{RESUMO}

Objetivo: Revisar e analisar quais as possíveis causas que levam a não adesão a imunização no Brasil. Revisão bibliográfica: A vacinação é reconhecida como uma prática de intervenção que previne doenças com grande potencial de causar morbidades e mortalidade em massa. Entretanto, posturas comportamentais, como a promoção de ideias antivacina e falta de conscientização da população mais jovem acerca da gravidade das doenças imunopreveníveis, são alguns dos fatores que atualmente contribuem para a queda das taxas de imunização e o retorno de doenças até então erradicadas. Considerações finais: Este constructo é de fundamental importância, pois irá prover embasamento teórico-metodológico para a comunidade acadêmica e científica sobre as possíveis motivações da recusa populacional quanto à adesão da imunização e a análise da cobertura vacinal no Brasil. Ademais, por meio da análise dos fatores que levam a população a não se vacinar, possibilitará a construção de debates que sustentem o planejamento de estratégias para impulsionar a adesão à imunização.

Palavras-chave: Cobertura vacinal, Antivacina, Imunização.

\begin{abstract}
Objective: To review and analyze the possible causes that lead to non-adherence to immunization in Brazil. Bibliographic review: Vaccination is recognized as an intervention practice that prevents diseases with great potential to cause mass morbidity and mortality. However, behavioral attitudes, such as the promotion of antivaccine ideas and lack of awareness among the younger population about the severity of vaccine-preventable diseases, are some of the factors that currently contribute to the fall in immunization rates and the return of diseases that had been eradicated. Final considerations: This construct is of fundamental importance because it will provide a theoretical and methodological basis for the academic and scientific community about the possible motivations for population refusal regarding adherence to immunization and the analysis of vaccination coverage in Brazil. In addition, through the analysis of the factors that lead the population not to be vaccinated, it will enable the construction of debates that support the planning of strategies to boost adherence to immunization.
\end{abstract}

Key words: Vaccination coverage, Anti-vaccine, Immunization.

\footnotetext{
${ }^{1}$ Faculdade Santo Agostinho (FASA), Vitória da Conquista - BA. *E-mail: shesllengta@hotmail.com

2 União Educacional do Vale do Aço (UNIVAÇO), Ipatinga - MG.

3 Universidade Estadual de Montes Claros (UNIMONTES), Montes Claros - MG.
}

SUBMETIDO EM: 3/2021

ACEITO EM: 3/2021

PUBLICADO EM: 3/2021 


\section{RESUMEN}

Objetivo: Revisar y analizar las posibles causas que conducen a la falta de adherencia a la inmunización en Brasil. Revisión bibliográfica: La vacunación es reconocida como una práctica de intervención que previene enfermedades con gran potencial de causar morbilidad y mortalidad masivas. Sin embargo, las actitudes conductuales, como la promoción de ideas antivacunas y la falta de conciencia entre la población más joven sobre la gravedad de las enfermedades prevenibles por vacunación, son algunos de los factores que actualmente contribuyen a la caída de las tasas de inmunización y al regreso de enfermedades. que hasta ahora había sido erradicado. Consideraciones finales: Este constructo es de fundamental importancia porque proporcionará una base teórica y metodológica para la comunidad académica y científica sobre las posibles motivaciones del rechazo de la población en cuanto a la adherencia a la inmunización y el análisis de la cobertura de vacunación en Brasil. Además, a través del análisis de los factores que llevan a la población a no vacunarse, permitirá la construcción de debates que apoyen la planificación de estrategias para impulsar la adherencia a la inmunización.

Palabras clave: Cobertura de vacunación, Anti-vacuna, Inmunización.

\section{INTRODUÇÃO}

A vacinação é reconhecida como uma prática de intervenção que previne doenças com grande potencial de causar morbidades e mortalidade em massa. Além de proteger os indivíduos vacinados, a prática acaba protegendo indiretamente os não vacinados, sendo capaz de eliminar a circulação de agentes infecciosos no meio ambiente e, consequentemente, conferir proteção a indivíduos vulneráveis (BARBIERI CLA, et al., 2017).

Considerando o cenário nacional, temos que há a construção de iniciativas públicas, ao longo das gestões político-administrativas, que buscam promover e conscientizar os benefícios da vacinação. Um exemplo disso, são as ações de vigilância epidemiológica e controle da vacinação que foram implementadas por meio da criação do Programa Nacional de Imunização (PNI) e institucionalizadas pela Lei 6.259 de 30 de outubro de 1975 e pelo Decreto № 78.231 de 12 de agosto de 1976 (LIMA AA e PINTO EDS, 2017).

O PNI traz como principal meta a vacinação de pessoas vivendo em território brasileiro em todas as fases da vida. Além disso, conduz por meio de planejamento em saúde, estratégias de prevenção, controle e promoção à saúde que influenciam de modo significativo na redução de hospitalizações, morbidades relacionadas a doenças e consequentemente ampliação da expectativa de vida (LIMA AA e PINTO EDS, 2017).

Atualmente, o PNI é tido como referência mundial pela adoção de estratégias diferenciadas para garantir alta cobertura, como a vacinação de rotina, campanhas anuais de vacinação, estabelecimento de metas, ampliação da população-alvo (integrando a vacinação para crianças, adolescentes, adultos e idosos), e por sua política de parcerias e incentivo a ampliação e modernização tecnológica da produção nacional de imunobiológicos (PEREIRA DDS, et al., 2013).

Nesta perspectiva, esse programa nacional se torna uma ferramenta capaz de conferir imunidade gratuita contra mais de quinze tipos diferentes de patógenos, provocando uma crescente cobertura desde a década de 90, indicando uma adesão satisfatória da população. Contudo, no ano de 2016 a cobertura vacinal iniciou um declínio importante de cerca de 10 a 20 pontos percentuais, ocasionando concomitantemente aumento da taxa de mortalidade infantil e materno e surto de transmissão de sarampo. Para mais, as principais causas deste declínio estão relacionadas principalmente com aspectos culturais e sociais, técnicos, movimentos antivacinas e o próprio enfraquecimento do Sistema Único de Saúde (SUS) (SATO APS, 2017).

Segundo dados do Departamento de Informática do Sistema Único de Saúde (DATASUS), de 1994 a 1999 observou-se um aumento da cobertura vacinal, entre os anos 2000 e 2014, tornando o cenário estável. Além disso, o ano de 2015 surge como um destaque nesse cenário, pois a cobertura de vacinação chegou a $95,07 \%$, sendo a maior nos últimos 24 anos. Por outro lado, ao avaliar os anos de 2016, 2017 e 2018, houve 
um decréscimo significativo durante o ano de 2016. Nesse período o Brasil apresentou uma porcentagem de $50,44 \%$ de cobertura, no ano de 2017 um valor correspondente a $39,76 \%$ e a menor taxa observada foi no ano de 2018 apresentando 34,42\% em todo país (BRASIL, 2020).

Partindo desses pressupostos, considera-se que a prática de vacinação é uma medida de grande importância para a saúde pública, pois é capaz de reduzir substancialmente as taxas de mortalidade e morbidade. Apesar de ser uma prática conferida a todos os brasileiros por meio da regulamentação pelo PNI e, à vista disso, ser amplamente difundida pelo território nacional, observa-se uma queda nas taxas de vacinação por diferentes motivos e isto tem causado preocupação as secretarias de vigilância epidemiológica (COUTO MT e BARBIERI CLL, 2015).

Diante do exposto, o presente constructo possui como objetivo de revisar e analisar quais as possíveis causas que levam a não adesão a imunização no Brasil.

\section{REVISÃO BIBLIOGRÁFICA}

O ano de 1796 foi marcado pelo surgimento da varíola na região da rota da seda localizada entre a China e a Turquia. Neste contexto, o médico inglês Edward Jenner propôs retirar o pus produzido por um doente contaminado pela doença e inocular em um indivíduo saudável. O médico percebeu que os indivíduos saudáveis ao desenvolverem sintomas benignos estavam protegidos da infecção considerada letal e denominou a técnica com o nome variolização, sendo essa a única vacina existente nos 90 anos subsequentes (DURÃES FAA, et al., 2019).

Apesar desta grande descoberta, somente no final do século XIX após a compreensão da dinâmica dos microrganismos por Louis Pasteur, foi que a primeira vacina, contra a raiva, foi testada em uma mulher que havia sido mordida por um cão infectado. O vírus dessa doença pertence ao gênero Lyssavirus e pode contaminar diversos mamíferos, ocasionando edemas em órgãos vitais como o cérebro e, consequentemente, comprometimento de todo o sistema nervoso central do indivíduo. Apesar da gravidade dessa infeção, esta mulher sobreviveu e a partir de então as pesquisas para o desenvolvimento de vacina contra diversas outras doenças foram alavancadas (HENRIQUES IJDS, et al., 2018).

A introdução da vacina no Brasil foi viabilizada pelo Barão de Barbacena no ano de 1840 que conferiu proteção inicialmente a famílias de nobres da época. Após 24 anos, a então capital do Rio de Janeiro foi assolada por epidemias de varíola, febre amarela e peste bubônica, somado a grandes instabilidades econômicas e precariedades de saneamento básico. A população foi imposta de maneira agressiva a aderir a vacinação e a partir disso instaurou-se o caos na cidade, dando origem a "Revolta da Vacina", ocasião em que a população se rebelou contra os gestores políticos, ateando fogo em estabelecimentos e apedrejando diversos locais da cidade (CRESCÊNCIO CL, 2017).

A recusa vacinal é um fenômeno que está presente desde o surgimento da primeira vacina, portanto os movimentos de defesa a liberdade individual e direito a escolher se vacinar ou não são manifestações especificamente contemporâneas. Os indivíduos adeptos ao movimento antivacina questionam a segurança dos imunobiológicos e seus possíveis efeitos colaterais (FRANCO MAE, et al., 2020).

Esse movimento ganhou força no início de 1998 após a publicação de um artigo na revista The Lancet, na qual o médico Dr. Andrew Wakefield e colaboradores afirmaram a relação entre o autismo e a vacina tríplice viral. Entretanto, o General Medical Council publicou uma declaração descrevendo a atitude antiética e irresponsável dos autores que fizeram a publicação, com isso o Dr. Andrew Wakefield foi criminalmente responsabilizado, teve o registro médico caçado e o artigo foi retirado dos arquivos do periódico (COSTA BBD, et al., 2020).

Assim, após sair a publicação na revista The Lancet as pessoas ficaram com receio de tomar vacinas com medo de desenvolver autismo e com medo dos possíveis efeitos colaterais dos componentes das vacinas. $\mathrm{E}$ mesmo após a declaração de que o artigo não apresentava informações confiáveis e após sua retirada da revista The Lancet, o movimento antivacina ganhou ainda mais força. Nos dias atuais a relação do autismo 
com a vacinação não é tão grande como no passado, mas os adeptos ao movimento antivacina afirmam que as vacinas podem conter substancias potencialmente toxicas e colocam em cheque a credibilidade delas, já que as indústrias lucram muito em cima da produção das mesmas (COSTA BBD, et al., 2020).

Somente no ano de 1976 foi publicada a regulamentação que detalha a forma como a vacinação obrigatória deveria ser realizada em território brasileiro, por meio da lei ํㅜ 6.259/75 e do Decreto ํㅜ 78.231/76. $O$ decreto afirma que a partir do dado ano de publicação todas as pessoas vivendo em território nacional brasileiro devem ser vacinados segundo as recomendações do Ministério da Saúde contra doenças e patógenos que tem potencial de controle epidemiológico pela vigilância sanitária. Dispõe ainda que os indivíduos menores de idade também devem ser vacinados e a dispensa à vacinação somente será permitida mediante apresentação de atestado médico justificando a contraindicação (CRESCÊNCIO CL, 2017).

Além disso, o Estatuto da Criança e do Adolescente (ECA), apresenta a Lei 8.069/90, que prevê diversas normas com objetivo de proteger o direito à vida e à saúde de crianças e adolescentes. Entre elas, há a previsão de punições aos pais que não vacinarem os filhos. A legislação afirma que "é obrigatória a vacinação das crianças nos casos recomendados pelas autoridades sanitárias", bem como as vacinações da primeira infância (CRESCÊNCIO CL, 2017; BRASIL, 1990).

A vacinação universal e igualitária em território brasileiro foi regulamentada ainda pelo PNI, criado em 1973 com o objetivo de controlar a infecção por doenças e reduzir mortalidade infantil (LESSA SDC e SCHRAMM FR, 2015). Este serviço de imunização, por meio do SUS viabiliza o acesso da população e propicia vacinação segura contra doenças imunopreveníveis. Além disso, por meio da elaboração de políticas, estratégias, supervisão e elaboração, dispõe-se a manutenção a nível de Atenção Primária à Saúde (APS), permitindo a ampliação da atenção integral à saúde (SIQUEIRA LDG, et al., 2015).

Durante muitos anos, doenças comuns na infância, como a meningite, difteria, poliomielite, hepatite B, rotavírus, febre amarela, caxumba, rubéola, sarampo coqueluche e tétano foram responsáveis por taxas elevadas de morbimortalidade em crianças menores de cinco anos em todo o mundo, principalmente nos países em desenvolvimento. Apesar disso, no Brasil, as taxas de óbito infantil reduziram-se substancialmente visto que após a implementação do PNI, quase $100 \%$ da população infantil brasileira foi imunizada contra as principais doenças responsáveis por óbito precoce. Ademais, o PNI oferece cobertura vacinal ainda para a população adulta em todas as fases da vida, sem distinção de caráter, de modo a controlar e prevenir doenças com potencial de letalidade (CAVALCANTI MAF e NASCIMENTO EGC, 2015).

Nesta perspectiva, esse programa nacional se torna uma ferramenta capaz de conferir imunidade gratuita contra mais de quinze tipos diferentes de patógenos, provocando uma crescente cobertura desde a década de 1990, indicando uma adesão satisfatória da população. Contudo, no ano de 2016 a cobertura vacinal iniciou um declínio importante de cerca de 10 a 20 pontos percentuais, ocasionando concomitantemente aumento da taxa de mortalidade infantil e materno e surto de transmissão de sarampo. Para mais, as principais causas deste declínio estão relacionadas principalmente com aspectos culturais e sociais, aspectos técnicos, movimentos antivacinas e próprio enfraquecimento do SUS (SATO APS, 2017).

O Brasil é conhecido mundialmente pelo seu amplo espectro de imunizações, que é distribuída gratuitamente a toda população brasileira. Entretanto, desde 2013 a queda das taxas de imunização vem caindo no país, principalmente dos imunobiológicos para caxumba, sarampo e rubéola, isso tem aumentado o risco da volta de surtos e epidemias dessas doenças. Alguns dos possíveis fatores é a falta de estrutura das cidades para gerir o sistema de imunização e a recusa dos pais a não levarem seus filhos para vacinarem (BARATA RB, et al., 2012).

Além disso, a falta de vínculo com as Unidades de Saúde é uma das razões pelas quais houve um decréscimo na cobertura de imunização, isso acontece porque há falta de contato entre pacientes e profissionais de saúde, acabando por dificulta as ações de promoção e prevenção. Ainda, um dos possíveis fatores da queda da imunização é a falta de tempo que os pais têm para ir as Unidades de Saúde vacinar seus filhos, visto que o horário de funcionamento da Unidade não é acessível para algumas pessoas (BARATA $\mathrm{RB}$, et al., 2012). 
Ademais, tem muitas mães que atrasam as vacinas por receio de que as crianças venham a sentir dor ou apresentar efeitos adversos. Da mesma forma, outras pessoas deixam de se vacinar ou até mesmo divulgam informações pseudocientíficas por terem tido experiências traumáticas relacionadas a imunização. Também, existem profissionais homeopatas que ainda contraindicam o uso da vacina, mesmo não havendo unanimidade quanto a este assunto, muitos deles argumentam que o efeito maléfico da vacina é muito mais significativo do que o seu efeito benéfico, e também que os medicamentos homeopáticos tornam os seus pacientes imunes a doenças infecciosas (FERREIRA AV, et al., 2017).

Magalhães ACB, et al. (2017) realizaram um estudo prospectivo no qual analisaram o impacto do esquema de vacinação antipneumocócica entre pacientes idosos e com comorbidades na cidade de São Paulo entre junho de 2015 e junho de 2016. Foram entrevistados 68 pacientes com idade superior a 60 anos, destes, $75 \%$ nunca haviam recebido qualquer tipo de orientação acerca da importância da vacina antipneumocócica. Os autores afirmam que isto é preocupante, pois essa imunização previne contra as formas invasivas de infecção pneumocócica, sendo a população idosa um grupo altamente vulnerável devido as grandes chances de evoluírem para complicações como meningite e bacteremia.

No cenário nacional, registrou em 2016 o menor número de imunizações no país, 84\%, sendo que o recomendado pela OMS é de $95 \%$. O risco dessa queda é a criação de "bolsões de pessoas suscetíveis" a doenças que já foram erradicadas no país. Porque apenas algumas pessoas infectadas, junto com pessoas não imunizadas pode gerar um surto de doenças antigas que já foram controladas (CRUZ A, 2017).

Um dos possíveis motivos que pode ter conduzido o Brasil ao cenário atual de surtos de doenças antigas que já tinham sido controladas é o seu próprio sucesso nas imunizações. Muitos pais entre 30 e 50 anos, que não viveram a realidade das epidemias de sarampo e poliomielite, não acompanharam de perto essas doenças e cresceram com a percepção de que a vacinação não compõe parte fundamental da imunização da população. Ademais, a falta de campanhas de vacinação com informações sobre a importância e os benefícios da vacinação nas mídias como televisão e redes sociais tem contribuído para a queda das taxas de imunização (LIMA NL, et al., 2016).

O PNI tem uma alta cobertura de imunizações que são oferecidos gratuitamente à população, entretanto, a estrutura de algumas cidades para receber esses imunobiológicos é precária (HOMMA, et al., 2020). É comum as Unidades de Saúde não terem geladeira adequada para armazenamento, em algumas regiões do país faltam insumos para aplicação das vacinas. Além disso, muitos técnicos de enfermagem trocam os imunobiológicos que irão ser aplicados, contribuindo para que haja um aumento nos erros de aplicações de vacinas. Dessa forma, isso acaba por gerar imunização biológica inadequada, aumento dos custos, redução da confiança da população em relação aos programas de vacinação e também acaba por fortalecer as ideias da população antivacina (OLIVEIRA VCD, et al., 2019).

Assim, é importante manter a segurança em todo processo de produção, distribuição, armazenamento e aplicação das vacinas. O próprio PNI tem o programa chamado "vacina segura", que tem como objetivo, principalmente na parte estrutural, a conservação de imunobiológicos para que nenhuma vacina perca a qualidade e não onere o sistema de saúde com o descarte de vacinas. Medidas como essas são importantes para gerar mais segurança para a população em relação à imunização (OLIVEIRA VCD, et al., 2019).

Percebe-se que a internet é capaz de modificar as percepções espaço-temporal, promovendo uma mudança na dinâmica do campo social (LIMA NL, et al., 2016). Apesar de ser considerado um bom veículo para informações, as redes sociais são capazes de propagar rapidamente informações de baixa confiabilidade, como a ineficácia de vacinas, a sua inutilidade e perigos relacionados a esta prática. Nesse cenário, o compartilhamento de percepções equivocadas torna-se preocupante, pois muitas pessoas acessam as redes buscando notícias e conhecimento em saúde que impactam diretamente na tomada de decisões (KATA A, et al., 2012).

Dessa forma, temos que 0 acesso a informações em saúde adquiridas nas redes sociais são potenciais riscos para o crescente movimento antivacina. A velocidade em que as informações são transmitidas pela internet facilita a disseminação de Fake News e a desinformação acaba por se tornar comum, sendo 
conhecida por "zumbificação da informação" em que os indivíduos absorvem e disseminam notícias falsas ou distorcidas sem perceber. Com isso, a irresponsabilidade ocorre quando as pessoas não fazem uma análise crítica sobre o conteúdo que estão consumindo e não checam se as fontes de leitura são confiáveis (COSTA BBD, et al., 2020).

Assim, esses espaços de interação online estimulam leituras que são deveras descuidadas, apressadas e desconectadas, promovendo conhecimento superficial e aprendizado vago. É essencial ressaltar ainda que a troca de informações entre os indivíduos interconectados influencia também na troca de informações imprecisas, permitindo que o conhecimento natural e fundamentado seja substituído por estoques virtuais pouco profundos (LIRA J, et al., 2017).

Ademais, todas essas características influenciam também na mobilização de movimentos sociais, inclusive, movimentos antivacinação, que se manifestam por meio de uma enorme gama de atitudes que influenciam a hesitação à vacina. As pessoas buscam fontes online e se expõe a crenças culturais e opiniões que possam se assimilar com as suas próprias, reiterando decisões contrárias à imunização. Por isso, é importante ponderar as informações absorvidas na internet e combater o sistema de desinformação baseado em notícias sem embasamento científico (TOMENY TS, et al., 2017).

A confiança em uma vacina varia ao longo da sua introdução no sistema de vacinação, quando uma vacina é introduzida para combater um tipo de doença que está ocasionando uma alta morbimortalidade, a tendência é as pessoas estarem mais dispostas para aceitarem, com isso, a cobertura vacinal aumenta e ocorre uma queda da incidência da doença. Entretanto, quando ocorre um aumento no número de imunizações, também aumenta o número de efeitos colaterais, assim, a cobertura vacinal entra em declínio. Desta forma, aumenta a hesitação vacinal e pode ocorrer surtos de doenças que são previsíveis com o uso da vacina (FRANCO MAE, et al., 2020).

Assim, o controle de doenças transmissíveis está atrelado diretamente a adesão da população as imunizações, pois quando o indivíduo toma uma vacina ele não está apenas se imunizando, ele acaba por também levar proteção para o lugar em que vive, a chamada "imunidade em rebanho" (LIMA AA e PINTO EDS, 2017). E a proteção coletiva só ocorre se $80 \%$ da população for imunizada, por isso da ação conjunta de todos fazerem o seu papel de se imunizar tudo em prol do bem comum (ALVES SMC, et al., 2020).

Diante disso, nota-se que a imunização é uma importante estratégia que reduz significativamente as taxas de morbimortalidade, conferindo proteção a população de forma direta e indireta. Para mais, o PNI conta com uma grande credibilidade da comunidade científica, visto que oferece cobertura vacinal a toda a população brasileira em todas as fases da vida, além de proporcionar o aumento da expectativa de vida nos indivíduos. Por isso, a prática deve ser incentivada e a vigilância epidemiológica mantida, de modo a aumentar a cobertura em território nacional (MIZUTA AH, et al., 2019).

\section{CONSIDERAÇÕES FINAIS}

Mesmo se mostrando um problema muito grave e que pode gerar consequências permanentes na saúde dos indivíduos, esse tema apesar de não ser desconhecido, ainda não é tão abordado no meio cientifico, necessitando de mais estudos. Nesta perspectiva, a intensificação da adesão populacional ao movimento vacinal pode ser realizada por meio de um conjunto de ações governamentais planejadas, que utilizam artifícios como a indústria midiática e redes sociais buscando a desconstrução de ideologias, que não levam em conta um fundamento teórico-científico, responsável por deturpar o real propósito da imunização. Ademais, por meio da análise dos fatores que levam a população a não se vacinar, possibilitará a construção de debates que sustentem o planejamento de estratégias para impulsionar a adesão à imunização.

\section{REFERÊNCIAS}

1. ALVES SMC, et al. Vacinação: direito individual ou coletivo?. Cad. Ibero-amer., 2020; 9(3): 134-139. 
2. BRASIL. Lei no 8.069, de 13 de julho de 1990. Dispõe sobre o Estatuto da Criança e do Adolescente e dá outras providências.1990. Disponível http://www.planalto.gov.br/ccivil_03/leis/l8069.htm\#: :text=LEl\%20N\%C2\%BA\%208.069\%2C\%20DE\%2013\%20DE \%20JULHO\%20DE\%201990.\&text=Disp\%C3\%B5e\%20sobre\%20o\%20Estatuto\%20da,Adolescente\%20e\%20d\%C 3\%A1\%20outras\%20provid\%C3\%AAncias.\&text=Art.\%201\%C2\%BA\%20Esta\%20Lei\%20disp\%C3\%B5e,\%C3\%A0 \%20crian\%C3\%A7a\%20e\%20ao\%20adolescente.\&text=Nos\%20casos\%20expressos\%20em\%20lei,e\%20um\%20a nos\%20de\%20idade. Acessado em: 29 de março de 2021.

3. BARBIERI CLA, et al. A (não) vacinação infantil entre a cultura e a lei: os significados atribuídos por casais de camadas médias de São Paulo, Brasil. Cad. Saúde Pública, 2017; 33(2): 1-11.

4. BARATA RB, et al. Desigualdades socioeconômicas e cobertura vacinal: resultados de uma pesquisa de cobertura vacinal em 27 capitais brasileiras, 2007-2008. J Epidemiol Community Health, 2012; 66: 934-941.

5. BRASIL. Informações de saúde, assistência à saúde, imunizações, cobertura. Brasília: Ministério da Saúde. Datasus; 2020. Disponível em: http://www2.datasus.gov.br/DATASUS/index.php?area=02. Acesso em: 05 de abril de 2020.

6. COUTO MT, BARBIERI CLL. Cuidar e (não) vacinar no contexto de famílias de alta renda e escolaridade em São Paulo, SP, Brasil. Ciênc. saúde coletiva, 2015; 20(1): 105-114.

7. COSTA BBD, et al. O movimento antivacina no YouTube nos tempos de pós-verdade: Educação em saúde ou desinformação?. Rev. Mídia e Cotidiano, 2020; 14(1): 220-239.

8. CRESCÊNCIO CL. (não) vacinação infantil entre a cultura e a lei: os significados atribuídos por casais de camadas médias de São Paulo, Brasil. Cad. Saúde Pública, 2017; 33(2): 1-11.

9. CAVALCANTI MAF, NASCIMENTO EGC. Aspectos Intervenientes da criança, da família e dos serviços de saúde na imunização infantil. Rev. Soc. Bras. Enferm. Ped., 2015; 15(1): 31-7.

10. CRUZ, A. A queda da imunização no Brasil. Revista CONSENSUS, 2017; 7(3): 20-9.

11. DURÃES FAA, et al. Edward Jenner e a Primeira Vacina: estudo do discurso expositivo adotado em um Museu de Ciência. Rev. de História da Ciência, 2019; 7(4): 49-56.

12. FRANCO MA, et al. Causas da queda progressiva das taxas de vacinação da poliomielite no Brasil. Brazilian Journal of Health Review, 2020; 3(6): 18476-18486.

13. FRANCISCO PMSB, et al. Vacinação contra hepatite B em adolescentes residentes em Campinas, São Paulo, Brasil. Rev. bras. epidemiol., 2015; 18(3): 552-567.

14. FERREIRA AV, et al. Acesso à sala de vacinas nos serviços de atenção primária à saúde. Rev. Eletr. Enf., 2017; $21(38): 26-35$.

15. HENRIQUES IJDS, et al. Verdades e mitos das vacinas infantis no calendário de imunização nacional. Universo.edu., 2018; 10(2): 49-56.

16. HOMMA, et al. Vacinas e vacinação no Brasil: horizontes para os próximos 20 anos. Rio de Janeiro: Edições Livres, 2020; 244p.

17. KATA A, et al. Anti-vaccine activists, Web 2.0, and the postmodern paradigm - An overview of tactics and tropes used online by the anti-vaccination movement. Vaccine, 2012; 30(2): 3778-3789.

18. LIMA AA, PINTO EDS. O contexto histórico da implantação do Programa Nacional de Imunização (PNI) e sua importância para o Sistema Único de Saúde (SUS). Scire Salutis, 2017; 7(1): 53-62.

19. LESSA SDC, SCHRAMM FR. Proteção individual versus proteção coletiva: análise bioética do programa nacional de vacinação infantil em massa. Ciência saúde coletiva, 2015; 20(1): 1-11.

20. LIRA J, et al. A geração superficial: o que a internet está fazendo com os nossos cérebros. Gestão e tecnologia, 2017; $7(2): 124-129$.

21. LIMA NL, et al. As redes sociais virtuais e a dinâmica da internet. Gerais, Rev. Interinst. Psicol., 2016; 9(1): 90-109.

22. MAGALHÃES ACB, et al. Vacinação contra pneumonia em pacientes idosos portadores de comorbidades. Análise do impacto do esquema de vacinação antipneumocócica nos pacientes com mais de 60 anos. Rev Soc Bras Clin Med., 2017; 15(1): 33-38.

23. MIZUTA AH, et al. Percepção acerca da importância das vacinas e da recusa vacinal numa escola de medicina. Rev. paul. Pediatr., 2019; 37(1): 34-40.

24. OLIVEIRA VCD, et al. A percepção da equipe de enfermagem sobre a segurança do paciente em sala de vacinação. Rev. Cuidarte, 2019; 10(1): 11.

25. PEREIRA DDS, et al. Análise da taxa de utilização e perda de vacinas no programa nacional de imunização. Cad. Saúde Colet., 2013; 21(4): 420-4.

26. SATO APS. Qual a importância da hesitação vacinal na queda das coberturas vacinais no Brasil?. Rev Saúde Public., 2017; 37(1): 52-96.

27. SÁ TS, et al. O papel da vacina pneumocócica em idosos diabéticos. Revista de Medicina e Saúde de Brasília. 2018; 13(11): 278-284.

28. SIQUEIRA LDG, et al. Avaliação da organização e funcionamento das salas de vacina na Atenção Primária à Saúde em Montes Claros, Minas Gerais, 2015. Epidemiol. Serv. Saúde, 2015; 26(3): 557-568.

29. SILVEIRA B, et al. Atualização em poliomielite. Rev Med Minas Gerais, 2019; 29(13): 74-79

30. TOMENY TS, et al. Geographic and demographic correlates of autism-related anti-vaccine beliefs on Twitter, 200915. Social science e medicina, 2017; 191(7): 168-175.

31. ZORZANELLI RT, CRUZ MGA. Conceito de medicalização em Michel Foucault na década de 1970. Interface Comunicação Saúde e Educação, 2018; 66(22): 1-12. 